\title{
ON DOUBLY TRANSITIVE PERMUTATION GROUPS OF DEGREE PRIME SQUARED PLUS ONE
}

\author{
PETER J. CAMERON
}

(Received 5 January 1978)

Communicated by M. F. Newman

\begin{abstract}
Groups with the property of the title were considered by Chillag (1977); this paper completes his results by showing that, with known exceptions, they are triply transitive.
\end{abstract}

Subject classification (Amer. Math. Soc. (MOS) 1970): 20 B 20.

THEOREM A. Let $G$ be a 2-transitive permutation group of degree $p^{2}+1$, where $p$ is prime. Then one of the following occurs:

(a) $G$ is 3-transitive;

(b) $P S L\left(2, p^{2}\right) \leqslant G \leqslant P \Gamma L\left(2, p^{2}\right)$;

(c) $G$ is the Frobenius group of order 20, with $p=2$.

Thus, conclusion (d) of Chillag's Corollary (asserting that the stabilizer of two points has orbit lengths $\left.1,1,2(p-1),(p-1)^{2}\right)$ does not occur; or, more precisely, groups in case (d) occur already in case (b). This is a consequence of the following result.

THEOREM B. Let $G$ be a 2-transitive permutation group on $X$, of degree $n^{2}+1(n>1)$. Suppose that, for $x, y \in X, x \neq y, G_{x y}$ has orbit lengths $1,1,2(n-1),(n-1)^{2}$. Then $n=3, G=P S L(2,9)$ or $P \Sigma L(2,9)$.

Proof. The results of Higman (1970) imply that $G_{x}$ is a subgroup of $S_{n}$ wr $S_{2}$; so $G_{x}$ has an imprimitive subgroup $N(x)$ of index 2. For $y \neq x, K=N(x) \cap G_{y}$ has orbit lengths $1,1, n-1, n-1,(n-1)^{2}$, and the orbit of length $(n-1)^{2}$ is isomorphic (as $K$-space) to the direct product of the two orbits of length $n-1$. 
Now $N(y) \cap G_{x}=K^{\prime}$ is a subgroup of index 2 in $G_{x y}$ with the same orbit lengths as $K$. If $K \neq K^{\prime}$, then $K \cap K^{\prime}$ has four orbits of length $\frac{1}{2}(n-1)$, so the $K$-orbit of length $(n-1)^{2}$ splits into four orbits of length $\frac{1}{4}(n-1)^{2}$ of $K \cap K^{\prime}$. This is impossible since $\left|K: K \cap K^{\prime}\right|=2$. We conclude that

$$
N(y) \cap G_{x}=N(x) \cap G_{y} \leqslant N(x),
$$

whence $N(x)$ is strongly closed in $G_{x}$ with respect to $G$.

By the "two-graph transfer theorem" (see Taylor (1977), Theorem 6.1), either $G$ has a subgroup $N$ of index 2 with $N \cap G_{x}=N(x)$, or $G$ is an automorphism group of a non-trivial regular two-graph. In the first case, if $B$ is either orbit of length $n-1$ of $N_{x y}$, then $B \cup\{y\}$ is a block of imprimitivity for $N_{x}$, and so the setwise stabilizer $L$ of $B \cup\{x, y\}$ acts 2-transitively on it. Then

$$
|N: L|=\left(n^{2}+1\right) n^{2} /(n+1) n ;
$$

but this is not an integer for $n>1$.

In the second case we use the fact that, if $H$ is a rank 3 group whose parameters (in Higman's (1970) sense) are $k, l, \lambda, \mu$, and $G$ is a transitive extension of $H$ which acts on a regular two-graph, then $k=2 \mu$. (See Taylor (1977), Proposition 2.3.) Here $k=2(n-1), \mu=2$; so we have $n=3$. The rest of the theorem is clear.

\title{
References
}

D. Chillag (1977), “On doubly transitive permutation groups of degree prime squared plus one", J. Austral. Math. Soc. (A), 23, 202-206.

D. G. Higman (1970), "Characterization of families of rank 3 permutation groups by the subdegrees, I", Arch. der Math. 21, 151-156.

D. E. Taylor (1977), “Regular two-graphs", Proc. London Math. Soc. (3), 35, 257-274.

\author{
Merton College \\ Oxford OX1 4JD \\ England.
}

